\title{
Jaundice As a Presentation of Thyrotoxic Crisis
}

\author{
SHAH KHALED HABIB, ${ }^{1}$ MOHAMMAD ASHIKIMRAN KHAN, ${ }^{1}$ MD. SHAHRI AR MAHBUB, ${ }^{2}$ M ARASHID, ${ }^{3}$ MD. TITU \\ MIAH, ${ }^{4}$ RATAN DAS GUPTA, ${ }^{4}$ MD. BI LLAL ALAM ${ }^{5}$
}

\begin{abstract}
Thyrotoxic crisis is a life threatening condition which requires urgent detection and management. Due to its wide variety of presentation its diagnosis can be difficult in some cases and high index of suspicion is required for its diagnosis. We report a case of 45 year old lady presenting with fever, jaundice and passage of loose stool whose thyroid hormone profile proved her to be suffering from thyrotoxic crisis.
\end{abstract}

Keyword: Thyrotoxic crisis, Jaundice, Antithyroid drugs, Hypoxia

\section{Introduction}

Thyroid storm, also referred to as thyrotoxic crisis is defined as a severe and often life threatening exacerbation of thyrotoxicosis which is usually characterized by hyperthermia, tachycardia, severe agitation and altered mental status. ${ }^{1-3}$ In contrast jaundice is rare in such patients and generally occurs due to hepatic congestion from thyrotoxic heart failure or antithyroid drug therapy or hepatic necrosis from systemic embolization caused by atrial fibrillation or autoimmune/viral hepatitis or secondary biliary obstruction or primary biliary cirrhosis which may be associated with Grave's disease. ${ }^{4}$ In hyperthyroidism, liver function tests might reveal non-specific elevations of the transaminases, mild indirect hyperbilirubinemia (30\% of cases), alkaline phosphatase (ALP) elevations ( $40 \%$ of patients), and there may be minor changes in liver histology. ${ }^{3-4}$

Here we report a case of a 45 year old female with fever and jaundice who was diagnosed as thyrotoxic crisis where the cause could not be evaluated. We suggest patients with jaundice of unknown cause should have thyroid function tests performed as a part of their routine investigation.

\section{Case Report}

A 45 year old female was admitted with a history of low grade fever for 2 months with yellow coloration of sclera for 2 weeks and passage of frequent loose stool for the same duration. The patient had no history of chronic disease, pharmaceutical or herbal medication, blood transfusion, and contact with TB patient or traveling. On initial examination, the patient was febrile with recorded temperature of $100^{\circ} \mathrm{F}$, level of consciousness on Glasgow coma scale was 15, there was jaundice, the hands were tremulous and her BP and pulse rate were 110/60 $\mathrm{mm} \mathrm{Hg}$ and 120b/min and regular respectively. On 3rd day of admission, her level of consciousness on Glasgow coma scale became 8 , temperature was $105^{\circ} \mathrm{F}$ and the patient was tachypneic and lungs were clear. Her laboratory test showed CBC with ESR and peripheral blood picture were normal, serum creatinine $0.8 \mathrm{mg} / \mathrm{dl}$. Blood and urine culture were sterile. Liver function test showed serum bilirubin-10.85 mg/dl, SGPT-48U/L, ALP170U/L, prothrombin time-17sec. Screening for hepatitis A,B,C,E were negative. Ultrasound of the abdomen showed no abnormality. Her CXR P/A view, ECG and endoscopy of upper GIT were normal. Autoimmune markers such as antinuclear antibody were negative. Thyroid hormone levels showed:- TSH- $<0.25 \mathrm{mIU} / \mathrm{L}$ (N:0.3-5.0 mIU/L), T3-6nmol/L (N:1.23-3.54 nmol/L), T4-360 nmol/L (N:54-173 nmol/L). Thyroid scan and auto antibody tests could not be performed because of her moribund condition. The patient was treated as thyrotoxic crisis with antithyroid drugs, glucocorticoids and beta blocker. Unfortunately the patient expired on the 4th day of admission.

\section{Discussion}

Thyroid storm or crisis represents exaggerated manifestations of thyrotoxicosis. ${ }^{5}$ Cardinal features include fever, tachycardia (usually out of proportion to the degree of fever), central nervous system manifestations (varying from confusion to coma), and gastrointestinal dysfunction

1. Post-graduate trainee, Department of Medicine, Dhaka Medical College

2. FCPS (Medicine) course, Dhaka Medical College.

3. Professor, Department of Anatomy (CC), Bangladesh Medical College

4. Assistant Professor, Department of Medicine, Dhaka Medical College.

5. Associate Professor, Department of Medicine, Dhaka Medical College.

Correspondence: Dr. Mohammad Ashik Imran Khan, Post Graduate Trainee, Dhaka Medical College Hospital, Dhaka. E Mail: ashikmrn@yahoo.com 
with nausea, vomiting, or even jaundice in severe cases, which usually denotes a poor prognosis. ${ }^{6-7}$ Hyperglycemia, hypercalcemia , and LFT abnormalities are frequent findings ${ }^{7-8}$ A precipitating factor usually decompensates hyperthyroidism; this can be an infection, trauma, surgery, cerebrovascular accident, or even emotional stress. Most patients have obvious symptoms and signs of thyrotoxicosis and a history of untreated or partially treated hyperthyroidism. ${ }^{7,9}$

The co-occurrence of hyperthyroidism and abnormal liver function tests is rare and the mechanism underlying hepatic dysfunction is not well known. In etiology, enzyme induction and the role of venous congestion due to heart failure are stipulated. Hypoxia is known as another mechanism and it is anticipated that increased oxygen utilization cannot be totally compensated for by hepatic blood flow. In patients with high levels of T3 and T4, relatively severe hypoxemia develops and the pericentral parts of hepatic acini become prone to damage. The mechanism underlying hyperbilirubinemia is not well known either and there are no available data supporting the direct toxic effects of thyroid hormones on the liver. ${ }^{10}$

Jaundice can be diagnosed during the clinical course of thyrotoxicosis while mild increases are observed in ALT, AST and bilirubin levels. In a study by Thompson et al. abnormal function tests and, in particular, elevations of bilirubin were reported in hyperthyroidism. ${ }^{11}$

In thyrotoxicosis, the predominant cardiac complications are atrial tachyarrhythmias and heart failure. Atrial fibrillation is the most common, occurring in $10 \%$ to $22 \%$ of hyperthyroid patients. Heart failure leading to hepatic congestion causes clinically detectable jaundice. As well as atrial fibrillation produces thrombi which clogs the hepatic circulation and produces ischemic liver which manifests as jaundice. ${ }^{12}$ Studies assessing the incidence of embolic events in thyrotoxic patients who have atrial fibrillation have yielded conflicting information regarding the incidence of embolism .$^{13-14}$ In the largest retrospective study, it appears that thyrotoxic patients who have atrial fibrillation are not at greater risk for embolic events, compared with age-matched patients who have atrial fibrillation due to other causes.$^{14}$ The standard risk factors for embolic events in atrial fibrillation, including increased age and underlying heart disease, apply to thyrotoxic patients.

Antithyroid drugs have been reported to induce liver toxicity but usually in the form of acute hepatitis with elevation of parenchymal enzymes. ${ }^{15-16}$ The occurrence of acute cholestasis is rare, although it is associated more commonly with carbimazole than with propylthiouracil. Hepatotoxicity, mainly in the form of cholestasis, has been reported in six cases of carbimazole use and in one of propylthiouracil use. ${ }^{17,21,22}$ Only one study reported that cross reactivity of the two drugs caused hepatotoxicity. ${ }^{21}$

In Woeber's review previously published cases of patients who had developed hepatotoxicity following treatment with MMI and carbimazole were analyzed; 19 patients had cholestatic-form clinical presentations. Clinical improvement is slow, yet the condition is totally reversible. An analysis of the cases reported by Woeber demonstrated that advanced age and high-dose anti-thyroid medications were risk factors for cholestatic damage. ${ }^{23,24}$

The liver has an important role in metabolism of thyroid hormone, and autopsies have shown hepatic inflammation, fibrosis, and centrilobular necrosis in patients with hyperthyroidism. ${ }^{25}$ The pathogenesis of hepatic dysfunction is unknown; one theory suggests the liver is damaged by the systemic effects of excess thyroid hormone. Hyperthyroidism induces an increased metabolic rate, which is associated with increased oxidative capacity and oxidative damage of tissue. ${ }^{26}$

Thyroid hormones are also known to increase production of insulin-like growth factor within the liver and can cause changes in fatty acid and lipid synthesis. ${ }^{27}$ This hypermetabolic state makes the liver more susceptible to injury, and, in addition, thyroid hormones might also have a direct toxic effect on hepatic tissue.

Other associations between liver dysfunction and thyroid disease per se or its management are also well described. Autoimmune hepatitis is associated with autoimmune thyroid disease, and raised aminotransferase concentrations may be seen before thyroiditis is diagnosed. ${ }^{28}$ If these persist after correction of thyroid dysfunction, liver biopsy may be required to differentiate autoimmune hepatitis from liver involvement secondary to thyroid disease as immunosuppressive therapies are usually indicated for autoimmune hepatitis.

A strong association exists between Hashimoto's thyroiditis and primary biliary cirrhosis. ${ }^{29}$ In those affected, autoimmune thyroid failure might occur associated with the presence of thyroid microsome, thyroperoxidase, and thyroglobulin antibodies in addition to the presence of goitre. Patients with these antibodies or hypothyroidism (either clinical or biochemical), or both, may be found in a considerable number of those with primary biliary cirrhosis.

\section{Conclusion}

Thyroid hormone concentrations are important for normal hepatic function and metabolism of bilirubin. Lack of 
knowledge of the association between thyroid and liver abnormalities can lead to misdiagnosis, mistakes in management of patients, and consequent under-reporting of those patients affected. Published case series in those with hyperthyroidism show that results of liver tests can be severely abnormal and modest abnormalities are common. In certain individuals this can lead to serious morbidity and even mortality.

\section{Conflict of Interest: None}

\section{References:}

1. Rives J D, Shepard R M. Thyroid crisis. Am Surg 1951; $17: 406-18$

2. Waldstein S S, Slodki S L, Kaganiec G I, Bronsky D. A clinical study of thyroid storm. Ann Intern Med 1960; 52:626-42

3. Mazzaferri E L, Skillman T G. Thyroid storm: A review of 22 episodes with special emphasis on the use of guanethidine. Arch Intern Med 1969; 124:684-90

4. Villard J F, Tabarin A, Neau D, Boursier M L. Hyperthyroidism with severe intrahepatic cholestasis. Digestive diseases and Sciences 1999;44:10:2001

5. Burch HB, Wartofsky L. Life-threatening thyrotoxicosis: thyroid storm. Endocrinol Metab Clin North Am 1993; 22:263-277

6. Roth RN, McAuliffe MJ. Hyperthyroidism and thyroid storm. Emerg Med Clin North Am 1989; 7:873-883

7. Gavin LA. Thyroid crises. Med Clin North Am 1991; 75:179-193

8. de los Santos ET, Mazzaferri EL. Thyrotoxicosis. results and risks of current therapy. Postgrad Med J 1990; 87:277278,281-286,291-294

9. Nicoloff JT. Thyroid storm and myxedema coma. Med Clin North Am 1985; 69:1005-1017

10. Goldberg PA, Inzucchi SE. Critical issues in endocrinology. Clin Chest Med 2003;24:583-606

11. Pimental L, Hansen K. Thyroid disease in the emergency department: a clinical and laboratory review. J Emerg Med 2005;28:201-9

12. Ladenson P W. Recognition and management of cardiovascular disease related to thyroid dysfunction. Am J Med 1990; 88:638-41

13. Bar-Sela S, Ehrenfield M, Eliakim M. Arterial embolism in thyrotoxicosis with atrial fibrillation.Arch Intern Med 1981;141:1191

14. Peterson P, Hansen JM. Stroke in thyrotoxicosis with atrial fibrillation. Stroke 1988;19(1):15-8
15. Hanson JS. Propylthiouracil and hepatitis. Two cases and a review of the literature. Arch Intern Med 1984; 144: 994-6

16. Liaw YF, Huang MJ, Fan KD, Li KL, Wu SS, Chen TJ. Hepatic injury during propylthiouracil therapy in patients with hyperthyroidism. A cohort study. Ann Intern Med 1993; 118: 424-8

17. Ayensa C, Diaz de Otazu R, Cia JM. Carbimazole-induced cholestatic hepatitis. Arch Intern Med 1986; 146: 1455

18. Blom H, Stolk J, Schreuder HB, von Blomberg-van der Flier M. A case of carbimazole-induced intrahepatic cholestasis. An immune mediated reaction? Arch Intern Med 1985; 145: 1513-5

19. Dinsmore WW, O’Hara MD, Callender ME. Postanaesthetic carbimazole jaundice. N Engl J Med 1983; 309: 438

20. Ozenne G, Manchon ND, Doucet J, Hemet J, Schrub JC, Bercoff E. Carbimazole-induced acute cholestatic hepatitis. J Clin Gastroenterol 1989; 11: 95-7

21. Sadoul JL, Canivet B, Freychet P. Toxic hepatitis induced by antithyroid drugs: four cases including one with crossreactivity between carbimazole and benzylthiouracil. Eur J Med 1993; 2: 473-7

22. Seidman DS, Livni E, Ilie B, Blum I. Propylthiouracil-induced cholestatic jaundice. J Toxicol Clin Toxicol 1986; 24: 35360

23. Motomura K, Brent G. Mechanisms of thyroid hormone action. Endocrinol Metab Clin North Am 1998;27:1-23

24. Klein I, Ojama K. Thyroid hormone and the cardiovascular system. N Engl J Med 2001; 344(7): 501-8

25. Ohshima T, Maeda H, Takayasu T, Fujioka Y, Nakaya T, Saito K, et al. An autopsy case of sudden death due to hyperthyroidism. Nippon Hoigaku Zasshi 1990; 44: 365-70

26. Venditti P, Pamplona R, Ayala V, De Rosa R, Caldarone G, Di Meo S. Differential effects of experimental and cold induced hyperthyroidism on factors inducing rat liver oxidative damage. J Exp Biol 2006; 209: 817-25

27. Tada H, Watanabe Y, Futakuchi Y, Amino N. Change in serum concentration of insulin-like growth factor in patients with thyroid disease. Endocr J 1994; 41: 541-5

28. Nobili V, Liaskos C, Luigi G, Guidi R, Francalanci P, Marcellini M. Autoimmune thyroiditis associated with autoimmune hepatitis. Thyroid 2005; 15: 1193-5

29. Crowe JP, Christensen E, Butler J, Wheeler P, Doniach D, Keenan J, et al. Primary biliary cirrhosis: the prevalence of hypothyroidism and its relationship to thyroid autoantibodies and sicca syndrome 